\title{
Error Analysis of Recurrence Equations
}

\author{
By R. Tait
}

Abstract. An error analysis of the Miller algorithm for computation from threeterm recurrence equations is given. Bounds are supplied in terms of the known coefficients and a method of finding suitable starting values for prescribed relative error is investigated.

1. Introduction. The three-term recurrence relation

$$
u_{r-1}(x)=p_{r}(x) u_{r}(x)+q_{r}(x) u_{r+1}(x),
$$

with $u_{r}(x)$ a function of a positive integer $r$ and a real variable $x$, is widely used in computational procedures. Two starting values $u_{n+1}=\gamma, u_{n}=1$, say, are required for backward computation, an appropriate normalisation factor being determined later. Further, a computation based on (1.1) is frequently insensitive to the choice of $\gamma$; that is $k$ significant figures of the normalised sequence $u_{n-m}, u_{n-m-1} \cdots$ are reliable for $m$ sufficiently large.

The method of computation is simple and readily adaptable to digital computers, a single known value of $u_{r}$ permitting scaling of the computed values to yield a desired solution. Stegun and Abramowitz [8] have pointed out a second advantage; that it is sometimes possible to deduce a scaling factor from computed values alone. A discussion of convergence and error analysis may be found in Olver [6].

In this note we examine the possibility of obtaining simple error bounds in terms of the coefficients $p_{r}(x)$ and $q_{r}(x)$. In this way it is possible to specify a starting value $r=n$ to obtain a required accuracy. In the case of the Bessel functions $I_{r}(x)$ and $J_{r}(x)$ an investigation of this point has previously been made by Makinouchi [4] and Shintani [7]. The problem has now amassed a considerable literature and we do not attempt an exhaustive bibliography. Similar results on continued fractions are to be found in Khovanski [3] and Khinchine [2].

Cases of (1.1) in which two solutions increase at equal rates are excluded; computationally this is a simple case. We restrict our attention to the case where (1.1) may be written as

$$
u_{r-1}=p_{r} u_{r}+q_{r} u_{r+1},
$$

with $p_{r}>0$ for values of $r$ considered. Of course, if $p_{r}<0$ for all $r$ then we would set

$$
v_{r}=(-1)^{r} u_{r}
$$

General expressions and bounds for the error term are developed in Section 2 and special cases considered in Sections 3 and 4. Our main interest is to examine the error. We do not directly examine convergence of the procedure; references to this question may be found in [6]. Nor do we consider improving the rate of con-

Received September 8, 1966. Revised December 6, 1966. 
vergence of the procedure, although it will become clear from our results when it is advantageous to do so.

2. Error in Exact Arithmetic. Eq. (1.2) in the form

$$
u_{r-1}=p_{r} u_{r}+q_{r} u_{r+1},
$$

is suitable for backward computation, and we concentrate our attention on this form, ignoring round-off error for the time being. We assume $p_{r}, q_{r}$ defined for $1 \leqq r \leqq n$ and any $x$ considered, with $q_{r} \neq 0$. By a nondecreasing solution of (2.1) we mean that the solution is nondecreasing as $r$ decreases. We denote the wanted solution by $w_{r}$, and assume that $w_{r}$ is positive, nondecreasing for values of $r$ considered. If $f_{r}$ and $g_{r}$ denote solutions of (2.1) such that $f_{n+1}=0, f_{n}=1$, and $g_{n+1}=1$, $g_{n}=0$, then, since $q_{r} \neq 0, f_{r}, g_{r}$ form a fundamental system of solutions, (Fort [1]), and we have

(i) $f_{r}, g_{r}$ are linearly independent for $0 \leqq r \leqq n+1$,

(ii) $w_{r}=w_{n} f_{r}+w_{n+1} g_{r}$.

In practise (2.1) is used with starting values $u_{n+1}=\gamma, u_{n}=1$, where we restrict $0 \leqq \gamma<1$. A normalisation constant $\eta$ is then required and we assume a value of $w_{r}$ known which we take to be $w_{0}(\neq 0)$. Then, provided $f_{0}+\gamma g_{0} \neq 0, \eta$ is given by

$$
\eta\left(f_{0}+\gamma g_{0}\right)=w_{0} .
$$

It is usual to take $\gamma=0$, but convergence of the procedure may be increased with a nonzero $\gamma$; see below.

Introducing the notation

$$
\begin{gathered}
E_{r}=\eta\left(f_{r}+\gamma g_{r}\right)-w_{r}, \quad E_{0}=0, \text { and } \\
E_{r}^{*}=E_{r} / w_{r}, \quad w_{r} \neq 0,
\end{gathered}
$$

for the error and relative error, respectively, we have

Theorem 2.1. If $f_{r} \neq 0,0 \leqq r \leqq n$, and $f_{0}+\gamma g_{0} \neq 0$, then for $1 \leqq r \leqq n$,

$$
E_{r}=(-1)^{n-r} \frac{\left(w_{n+1}-\gamma w_{n}\right)}{\left(f_{0}+\gamma g_{0}\right)} f_{r} f_{0} \sum_{s=0}^{r-1}(-1)^{s} \frac{q_{r-s} \cdots q_{n}}{f_{r-s} f_{r-s-1}} .
$$

Proof. Assume $w_{n+1} \neq \gamma w_{n}$, otherwise the result is trivial. We have

$$
\eta\left(f_{0}+\gamma g_{0}\right)=w_{n} f_{0}+w_{n+1} g_{0},
$$

giving

$$
E_{r}=\frac{\left(w_{n+1}-\gamma w_{n}\right)}{\left(f_{0}+\gamma g_{0}\right)} f_{0} f_{r}\left(\frac{g_{0}}{f_{0}}-\frac{g_{r}}{f_{r}}\right)
$$

But

$$
\frac{g_{r}}{f_{r}}=\frac{g_{r-1}}{f_{r-1}}+(-1)^{n-r+1} \frac{q_{r} \cdots q_{n}}{f_{r} f_{r-1}},
$$

and the result follows by induction.

We note the following for future reference.

Corollary 2.1. (i) $E_{r}$ satisfies $E q$. (2.1) 
(ii) If the sum in (2.5) is written $\sum_{s=0}^{r-1} T_{s}$, then

$$
\left|\frac{T_{s+1}}{T_{s}}\right|<1 \text { if and only if }\left|\frac{f_{r-s-2}}{f_{r-s}}\right|>\left|q_{r-s-1}\right| \text {. }
$$

(iii) If $\left|T_{s+1} / T_{s}\right| \leqq \alpha<1, s=0,1, \cdots, r-2$, then

$$
\left|E_{r}\right| \leqq(1-\alpha)^{-1}\left|\frac{w_{n+1}-\gamma w_{n}}{f_{0}+\gamma g_{0}} f_{r} f_{0} \frac{q_{r} \cdots q_{n}}{f_{r} f_{r-1}}\right| .
$$

We shall take $\gamma=0$ for simplicity, but it is clear from (2.5) how the value of $\gamma$ affects $E_{r}$. Again, with $R_{r}=E_{r} / f_{r}$, Eq. (2.6) shows the close connection between the present problem and convergents to continued fractions. The arguments which follow are abbreviated where similar material may be found in for example (2) or (3). We proceed to the simplest case, that of positive coefficients.

3. Positive Coefficients. Let us assume that $p_{r}>0, q_{r}>0$ for $1 \leqq r \leqq n$. Then $f_{r}>0$ and $g_{r}>0$. Use of (1.2) with $r$ replaced by $r-s-1$ shows that Corollary (2.1), (ii) holds. With these conditions it is clear that the terms in the sum in (2.5) are of alternating sign and decreasing magnitude, so that $E_{0}=0$,

$$
\begin{aligned}
& E_{1}=(-1)^{n-1} \frac{w_{n+1}}{f_{0}} \prod_{s=1}^{n} q_{s}, \\
& E_{2}=(-1)^{n-2} \frac{w_{n+1}}{f_{0}} p_{1} \prod_{s=2}^{n} q_{s},
\end{aligned}
$$

where in (3.2) we have used the recurrence relation to combine the two terms. In general

$$
0 \leqq p_{r-1} \frac{w_{n+1}}{f_{r-2}} \prod_{s=r}^{n} q_{s} \leqq(-1)^{n-r} E_{r} \leqq \frac{w_{n+1}}{f_{r-1}} \prod_{s=r}^{n} q_{s}, \quad 2 \leqq r \leqq n,
$$

for given fixed $n$. Clearly $E_{r}$ alternates in sign, and since $w_{r}=w_{n} f_{r}+w_{n+1} g_{r}$, we have

$$
0 \leqq(-1)^{n-r} E_{r}^{*} \leqq \frac{w_{n+1}}{w_{n}} \cdot \frac{1}{f_{r} f_{r-1}} \prod_{s=r}^{n} q_{s}, \quad r \geqq 2,
$$

with obvious expressions for $E_{1}{ }^{*}, E_{2}{ }^{*}$.

If further, $p_{r} \geqq 1$ for $r \geqq k$, say, where $k \geqq 1$, then $f_{r}, g_{r}$ are nondecreasing as $r$ decreases, for $k-1 \leqq r \leqq n$, and using simple induction we find that

$$
f_{r} f_{r-1} \geqq p_{n} \prod_{s=r}^{n-1}\left(p_{s} p_{s+1}+q_{s}\right), \quad k \leqq r \leqq n-1 .
$$

Substitution in the denominator of (3.4) yields

$$
0 \leqq(-1)^{n-r} E_{r}{ }^{*} \leqq \frac{\prod_{s=r}^{n} q_{s}}{p_{n+1} p_{n} \prod_{s=r}^{n-1}\left(p_{s} p_{s+1}+q_{s}\right)},
$$

provided $p_{n+1}>0$, for then $w_{n} \geqq p_{n+1} w_{n+1}$. (3.6) can be used either to find a bound for $E_{r}{ }^{*}$ or to find a suitable $n$ for specified bounds on $E_{r}{ }^{*}$. Again (3.6) supplies a strict upper bound using only known coefficients. Eq. (2.5) supplies the exact error, but its calculation would entail a knowledge of the values of all the $f_{r}$. 
The behaviour of $E_{r}, E_{r}{ }^{*}$ can be examined with the aid of the recurrence relation. We suppose that at stage $k$ a certain number of reliable figures, $m$ say, has been attained and we would like to be sure that this number persists for $r<k$. Since $E_{0}=0, E_{r}$ alternates in sign, and

$$
E_{r-1}=p_{r} E_{r}+q_{r} E_{r-1}
$$

we have

$$
\left|E_{2}\right|=\left(p_{1} / q_{1}\right)\left|E_{1}\right|
$$

and in general

$$
\left|E_{r}\right| \leqq\left(q_{r} / p_{r}\right)\left|E_{r+1}\right|
$$

so that if $p_{r} \geqq q_{r}>0$, then

$$
\left|E_{r}\right| \leqq\left|E_{r+1}\right|
$$

By our assumption that $w_{r}$ is nondecreasing and positive the same inequality holds for $E_{r}{ }^{*}$. Computed values of $f_{r}$ could be used to test whether there is a solution with the properties of $w_{r}$, or it would be sufficient, for example, that $p_{r} \geqq 1, r=1,2, \cdots$. The relevant material may be collected as follows.

THEOREM 3.1. (i) If $p_{r} \geqq q_{r}>0 ; 1 \leqq r \leqq n$, then for fixed $n$,

$$
\left|E_{r}\right| \leqq\left|E_{r+1}\right| \text { and }\left|E_{r}^{*}\right| \leqq\left|E_{r+1}^{*}\right|, \quad 1 \leqq r \leqq n-1 .
$$

(ii) If $p_{r}>0, q_{r}>0$ then

$$
\left|E_{r-1}^{*}\right| \leqq\left|E_{r+1}^{*}\right|, \quad 1 \leqq r \leqq n-1 .
$$

Proof. (i) follows from the discussion.

(ii) The alternating property of $E_{r}$ and relation (3.7) give

$$
\left|E_{r-1}\right| \leqq q_{r}\left|E_{r+1}\right|
$$

and so

$$
\left|E_{r-1}^{*}\right| \leqq q_{r} \frac{w_{r+1}}{w_{r-1}}\left|E_{r+1}^{*}\right| .
$$

Since $w_{r-1} \geqq\left(p_{r}+q_{r}\right) w_{r+1}$, by our assumption on $w_{r}$, we have

$$
\left|E_{r-1}^{*}\right| \leqq \frac{q_{r}}{p_{r}+q_{r}}\left|E_{r+1}^{*}\right| \leqq\left|E_{r+1}^{*}\right|
$$

Eq. (3.12) suggests that it would be advisable to examine the bound for $E_{r}{ }^{*}$ at least two successive points. We give two examples to illustrate these points.

Example 3.1. The Bessel Function $I_{r}(x)$.

$$
u_{r-1}(x)=(2 r / x) u_{r}(x)+u_{r+1}(x),
$$

with solutions $I_{r}(x),(-1)^{r} K_{r}(x)$. From (3.6) it follows that

$$
\left|E_{r}^{*}\right| \leqq \frac{(x / 2 n)^{2}}{\prod_{\substack{n=r \\ s=r}\left\{s(s+1) / x^{2}+1\right\}}, \quad r \geqq \frac{x}{2},}
$$

so that if say $x=100$ and $n=110$, then $\left|E_{100}^{*}\right| \leqq 2^{8} \times 10^{-10}$; with $x=100$ and $n=60,\left|E_{50}^{*}\right| \leqq 2^{-10}$. 
The improvement obtained by increasing $n$ is clear.

A simple example will show the practicality of our bounds. We take $x=2$ and $n=10$.

$\begin{array}{rccc}r & \text { Trial Values } & \text { Computed Values } & \text { Actual Values } \\ 11 & u_{11}=0 & - & - \\ 10 & u_{10}=1 & - & - \\ 9 & u_{9}=10 & (-6) 3.04389,0744 & (-6) 3.04418,5903 \\ 8 & u_{8}=91 & (-5) 2.76994,0577 & (-5) 2.76993,6951 \\ 7 & u_{7}=738 & (-4) 2.24639,1369 & (-4) 2.24639,1420 \\ 6 & u_{6}=5,257 & (-3) 1.60017,3364 & (-3) 1.60017,3364 \\ . & . & . & . \\ . & . & . & . \\ . & . & (0) 2.27958,5302 & \text { (0) } 2.27958,5302 \\ 0 & u_{0}=7,489,051 & & \end{array}$

with $\eta=3.04389,0744 \times 10^{-7}$

\begin{tabular}{|c|c|c|}
\hline$r$ & Bound for $\left|E_{r}{ }^{*}\right|$ & Actual $\left|E_{r}^{*}\right|$ \\
\hline 11 & - & - \\
\hline 10 & - & - \\
\hline 9 & (-4) 1.099 & $(-4) 0.9695$ \\
\hline 8 & (-6) 1.506 & $(-6) 1.309$ \\
\hline 7 & (-8) 2.641 & $(-8) 2.207$ \\
\hline 6 & $(-10) 6.142$ & - \\
\hline$\cdot$ & - & - \\
\hline - & - & - \\
\hline & - & - \\
\hline 0 & 0 & 0 \\
\hline
\end{tabular}

Example 3.2. Parabolic Cylinder Functions.

$$
u_{r-1}(x)=x u_{r}(x)+\left(r+\frac{1}{2}\right) u_{r+1}(x), \quad x \geqq 1 .
$$

Again, from (3.6) we have

$$
\left|E_{r}{ }^{*}\right|=\frac{\left(n+\frac{1}{2}\right)}{x^{2} \prod_{s=r}^{n-1}\left\{1+x^{2} /\left(s+\frac{1}{2}\right)\right\}},
$$

so that for $x=5, n=18$, say, we have $\left|E_{5}^{*}\right| \leqq 10^{-6}$. A little consideration shows that for fixed $r$ and $x,\left|E_{r}{ }^{*}\right|$ decreases as $n$ increases. If $x^{2}$ is of comparable magnitude to $n$, a factor of 2 enters the denominator for unit increase in $n$. If $x$ is small convergence will be slow.

4. $q_{r}$ Not Necessarily Positive. As is to be expected, this case is not so simple, nor convergence as rapid. If in (2.1), $p_{r}=p, q_{r}=q$, where $p$ and $q$ are constants such that $p>0, q<0$, and $p^{2}<4|q|$, then both solutions oscillate. The conditions below are sufficient to assure at least one positive monotone increasing solution for $r$ decreasing. We again assume $w_{r}$ has these properties. We take $\gamma=0$, and again assume $q_{r} \neq 0$. 
Taking $p_{r}>1+\left|q_{r}\right|, r=1,2, \cdots, n$, we are assured that $f_{r}$ is positive monotone increasing in the above sense. This condition is perhaps stronger than one would wish, but it avoids the need for calculating all the $f_{r}$ to investigate their monotonicity.

If $\alpha$ denotes the maximum value of $\left|T_{s+1} / T_{s}\right|$ in (2.5) then since

$$
\left|\frac{T_{s+1}}{T_{0}}\right|=\left|\frac{T_{1}}{T_{0}} \cdot \frac{T_{2}}{T_{1}} \ldots \frac{T_{s+1}}{T_{s}}\right|,
$$

we have

$$
\left|E_{r}\right| \leqq w_{n+1} / f_{r-1} \cdot\left|q_{r} \cdots q_{n}\right| \cdot\left(1+\alpha+\cdots+\alpha^{r-1}\right) .
$$

In fact, since

$$
\left|\frac{T_{s+1}}{T_{s}}\right|=\left|\frac{f_{r-s} q_{r-s-1}}{f_{r-s-2}}\right|,
$$

we can use the recurrence relation and the monotone increasing property of $f_{r}$ to show that

$$
\begin{aligned}
f_{r-s-2} & \geqq p_{r-s-1} f_{r-s-1}-\left|q_{r-s-1}\right| f_{r-s} \\
& \geqq\left(p_{r-s-1}-\left|q_{r-s-1}\right|\right) f_{r-s},
\end{aligned}
$$

and so, using the above expression for $\left|T_{s+1} / T_{s}\right|$, we find that $\alpha<1$, provided that

$$
\beta \equiv \max \left|\frac{q_{k}}{p_{k}-\left|q_{k}\right|}\right|<1, \quad k=1,2, \cdots, r-1 .
$$

Summarising, we have

Theorem 4.1. If $p_{k}>1+\left|q_{k}\right|, k=1,2, \cdots, n$, and

$$
p_{k}>\max \left\{1+\left|q_{k}\right|, 2\left|q_{k}\right|\right\}, \quad k=1,2, \cdots, r-1,
$$

then

$$
\left|E_{r}\right| \leqq w_{n+1} /(1-\alpha) f_{r-1} \cdot\left|q_{r} \cdots q_{n}\right|,
$$

where $\alpha$ is given by (4.3).

Complications are also added to the study of $E_{r}{ }^{*}$. However, with our conditions, it is readily verified that

$$
\left|E_{r}^{*}\right| \leqq \frac{w_{n+1}}{c_{n}(1-\alpha)} \cdot \frac{\left|q_{r} \cdots q_{n}\right|}{\prod_{\substack{n=r \\ s=2}}^{n-2}\left(p_{s}-\left|q_{s}\right|\right)},
$$

where

$$
c_{n}=\left(p_{n-1}-\left|q_{n-1}\right|\right) w_{n} p_{n}, \quad(n-r) \quad \text { even }
$$

and

$$
c_{n}=\left(p_{n} p_{n-1}-\left|q_{n-1}\right|\right) w_{n+1}, \quad(n-r) \quad \text { odd } .
$$

Clearly the bound (4.5) on $\left|E_{r}{ }^{*}\right|$ decreases as $r$ decreases for fixed $n$.

Example 4.1. Legendre Functions of the Second Kind, $Q_{n}(x)$.

$$
u_{r-1}(x)=(2+1 / r) x u_{r}(x)-(1+1 / r) u_{r+1}(x) .
$$


The preceding conditions hold certainly if $x>4 / 3$. Therefore

$$
\left|E_{r}^{*}\right| \leqq \frac{w_{n+1}}{c_{n}} \cdot\left(\frac{3 x-2}{3 x-4}\right) \cdot \frac{n}{n-2} \cdot \frac{1}{\prod_{s=r}^{n-2}((2 s+1) /(s+1) x-1)}, \quad n>2 .
$$

With $x=2, n=10$, we find that

$$
\left|E_{3}{ }^{*}\right| \leqq 3.5 \times 10^{-4} \text {, and }\left|E_{2}{ }^{*}\right| \leqq 7.1 \times 10^{-5} .
$$

With the aid of the tables [9], a short computation shows that

$$
\left|E_{3}{ }^{*}\right| \leqq 5.1 \times 10^{-6} \text {, and }\left|E_{2}{ }^{*}\right| \leqq 3.3 \times 10^{-6} \text {. }
$$

This calculation was carried out with $\eta=2.65720,05 \times 10^{-7}$, retaining four significant figures, and normalising by $Q_{0}(2)$.

If we wish to improve the bound for $\left|E_{3}{ }^{*}\right|$, that is, to find $n$ for a prescribed bound on $\left|E_{3}{ }^{*}\right|$, we proceed as follows. Let $\phi_{n}$ denote the above bound on $\left|E_{r}{ }^{*}\right|$ for starting value $n$, and assume, for simplicity, that $(n-r)$ is odd. Then we have

$$
\frac{\phi_{n+2}}{\phi_{n}}=\frac{\left(p_{n} p_{n-1}-\left|q_{n-1}\right|\right)}{\left(p_{n+2} p_{n+1}-\left|q_{n+1}\right|\right)} \cdot\left(1-\frac{4}{n^{2}}\right) \cdot \frac{1}{\prod_{s=n-1}^{n}((2 s+1) x /(s+1)-1)} .
$$

Thus, in the above example, if we increase $n$ to 12 , the bound is decreased by a factor of 0.13 , and by a further factor of 0.13 if we take $n=14$. It is now clear how to find $n$ for a prescribed bound on $\left|E_{j}^{*}\right|$.

Example 4.2. The Bessel Function $J_{n}(x)$.

$$
u_{r-1}(x)=(2 r / x) u_{r}(x)-u_{r+1}(x) .
$$

Our conditions hold only if $r>x$. This suggests normalisation at some point $r>x$. If $x$ is small, say 0.8 , then with $n=10$, and normalisation at $J_{0}$, we find

$$
\left|E_{3}{ }^{*}\right| \leqq 1.81 \times 10^{-10} \text {. }
$$

If one carries out the computation with a very accurate

$$
\eta=2.84368,81977,154 \times 10^{-11}
$$

one in fact finds

$$
\left|E_{3} *\right| \leqq 2.55 \times 10^{-15}
$$

for normalisation at $J_{0}(0.8)$.

Example 4.3. Coulomb Wave Function $F_{n}(\beta, x)$.

$$
u_{r-1}(\beta, x)=\frac{(2 r+1)[\beta+r(r+1) / x]}{(r+1)\left(r^{2}+\beta^{2}\right)^{1 / 2}} u_{r}(\beta, x)-\frac{r\left[(r+1)^{2}+\beta^{2}\right]^{1 / 2}}{(r+1)\left(r^{2}+\beta^{2}\right)^{1 / 2}} u_{r+1}(\beta, x) .
$$

For $r$ finite, $\left|q_{r}\right|<1$ so that the only condition we need verify is that $p_{r}>1+\left|q_{r}\right|$. In order to compare the present bounds with practical results, set $\eta=2, x=5$ as in [9, p. 543, Example 1]. This suggests normalisation at $u_{1}$. With $u_{r+1}$ replaced by $u_{r}$ (to coincide with our previous notation), our bound becomes

$$
\left.\left|E_{r}^{*}\right| \leqq \frac{6 w_{n+1}}{c_{n}} \cdot \frac{1}{\prod_{p=r}^{n-2}\left\{\frac{2 p-1}{5} \cdot \frac{[1+10 / p(p-1)]}{\left[1+(2 / p)^{2}\right]^{1 / 2}}-1\right\}}\right\} \quad \text { since } \alpha \leqq .83 .
$$


With $n=10$, we find that

$$
\left|E_{3}{ }^{*}\right| \leqq 3 \times 10^{-3} .
$$

Thus if we normalise by use of the value of $F_{1}(2,5)$, then $\left|E_{4}{ }^{*}\right| \leqq 3 \times 10^{-3}$, where $E_{4}{ }^{*}$ is the relative error in $F_{4}(2,5)$. Reference to the tables [9] and to [8] shows that

$$
\left|E_{4}{ }^{*}\right|=1.4 \times 10^{-3} \text {. }
$$

Finally we give an example due to Olver [6].

Example 4.4.

$$
u_{r-1}=\frac{12 r}{2 r-1} u_{r}-\frac{2 r+1}{2 r-1} u_{r+1}, \quad r \geqq 1 .
$$

Then $4 \leqq p_{r} /\left|q_{r}\right| \leqq 6$. For $n=4$ we find $\left|E_{2}{ }^{*}\right| \leqq 1 / 150$.

5. Round-Off Error. If $r=n$ gives the starting value, we consider the propagation of an error $\epsilon$ introduced at step $k(<n)$. For $r \leqq k$, we obtain $f_{r}+\delta_{r}$, say, rather than $f_{r}$ where

$$
\delta_{r}=A f_{r}+B g_{r}, \quad r \leqq k,
$$

and

$$
A=\frac{-\epsilon g_{k+1}}{f_{k+1} g_{k}-f_{k} g_{k+1}}, \quad B=\frac{\epsilon f_{k+1}}{f_{k+1} g_{k}-f_{k} g_{k+1}} .
$$

Denoting the new and old normalisation constants by $\eta_{1}$ and $\eta_{0}$, respectively, we have

$$
\eta_{1}\left(f_{0}+\delta_{0}\right)=\eta_{0} f_{0} .
$$

Again, if $E_{r}^{N}$ and $E_{r}$ denote the new and original errors, respectively, then

$$
E_{r}^{N}=\eta_{1} f_{r}-w_{r}, \quad r>k,
$$

and

$$
E_{r}^{N}=\eta_{1}\left(f_{r}+\delta_{r}\right)-w_{r}, \quad r \leqq k .
$$

For all $r$, set

$$
M_{r}=\eta_{1} f_{r}-w_{r}=\left(\eta_{1} / \eta_{0}\right) E_{r}+\left(\eta_{1} / \eta_{0}-1\right) w_{r},
$$

and

$$
M_{r}^{*}=M_{r} / w_{r}=\left(\eta_{1} / \eta_{0}\right) E_{r}^{*}+\left(\eta_{1} / \eta_{0}-1\right) .
$$

Since $E_{r}{ }^{*}$ has been previously bounded and

$$
\eta_{1} / \eta_{0}=\left(1+\delta_{0} / f_{0}\right)^{-1},
$$

we can use our previous ideas to find

$$
\frac{\delta_{0}}{f_{0}}=\epsilon_{k+1}\left\{\frac{1}{f_{k} f_{k+1}}+\sum_{s=1}^{k}(-1)^{s} \frac{q_{k+1-s} \cdots q_{k}}{f_{k+1-s} f_{k-s}}\right\},
$$

and so we may consider $M_{r}{ }^{*}$ known. For $r \leqq k$, we have still to consider the term $\eta_{1} \delta_{r}$. This is most conveniently carried out by noting that 


$$
\eta_{1}\left(f_{r}+\delta_{r}\right)-w_{r}=E_{r}+B \eta_{1} f_{r}\left(g_{r} / f_{r}-g_{0} / f_{0}\right) .
$$

Thus

$$
E_{r} *^{N}=E_{r}{ }^{*}+(-1)^{k-r} \epsilon\left(1+M_{r}^{*}\right) f_{k+1} \sum_{s=0}^{r-1}(-1)^{s} \frac{q_{r-s} \cdots q_{k}}{f_{r-s} f_{r-8-1}} .
$$

It is now clearly a matter of applying our preceding bounds to determine the magnitude of the additional error involved. As an illustration we repeat the straightforward Example 3.1 with $x=2$ and $n=10$.

Example 5.1. We write (5.9) as

$$
E_{r}{ }^{N}=E_{r}{ }^{*}+N_{r} .
$$

Then, with the above notation, and using the conditions of Section 3 , we have

$$
\left|N_{r}\right| \leqq\left(\frac{|\epsilon|}{f_{k}}\right) \cdot \frac{f_{k} f_{k+1}}{f_{r} f_{r-1}} \cdot \frac{\left(q_{r} \cdots q_{k}\right)\left(1+\left|E_{r}{ }^{*}\right|\right)}{\left(1-|\epsilon| / f_{k}\right)} .
$$

Suppose now that we replace the trial value 10 in $u_{9}$, and repeat the calculations. We tabulate these below, and use

$$
\left|N_{r}\right| \leqq \frac{10\left(1+\left|E_{r}^{*}\right|\right)}{9 p_{n} \prod_{s=r}^{n-1}\left(p_{s} p_{s+1}+q_{s}\right)},
$$

so that

$$
\left|E_{r} *^{N}\right| \leqq\left|E_{r}^{*}\right|+\left|N_{r}\right|
$$

$\begin{array}{rccc}r & \text { Trial Values } & \text { Computed Values } & \text { Actual Values } \\ 11 & 0 & - & - \\ 10 & 1 & - & - \\ 9 & 9 & (-6) 3.04022,8070 & (-6) 3.04418,5903 \\ 8 & 82 & (-5) 2.76998,5575 & (-5) 2.76993,6951 \\ 7 & 665 & (-4) 2.24639,0741 & (-4) 2.24639,1420 \\ 6 & 4,737 & (-3) 1.60017,3374 & (-3) 1.60017,3364 \\ . & \cdot & \cdot & \cdot \\ . & \cdot & \cdot & \cdot \\ . & . & \text { (0) } 2.27958,5302 & \text { (0) } 2.27958,5302\end{array}$

with $\eta=3.37803,1189 \times 10^{-7}$.

$\begin{array}{rcccc}r & \left|E_{r}{ }^{*}\right| \text { actual } & \left|N_{r}\right| \text { bound } & \left|E_{r}^{*}\right| \text { bound } & \left|E_{r}^{*}\right| \text { bound } \\ 11 & - & - & - & - \\ 10 & - & - & - & - \\ 9 & (-3) 1.3001 & (-3) 1.222 & (-4) 1.099 & (-3) 1.332 \\ 8 & (-5) 1.7554 & (-5) 1.673 & (-6) 1.506 & (-5) 1.824 \\ 7 & (-7) 3.0226 & (-7) 2.935 & (-8) 2.641 & (-7) 3.200 \\ 6 & (-9) 6.2493 & (-9) 6.825 & (-10) 6.142 & (-9) 7.440 \\ . & . & . & . & . \\ . & . & . & . & . \\ . & . & - & - & -\end{array}$


Finally, consider the alternative form of normalisation suggested by Olver [6],

$$
\eta_{1} \sum_{r=0}^{n} m_{r} f_{r}=1, \quad \text { where } \quad \sum_{r=0}^{\infty} m_{r} w_{r}=1 .
$$

We have

$$
\frac{\eta_{0}}{\eta_{1}}=\sum_{r=0}^{n} m_{r}\left(\eta_{0} f_{r}\right)=1-\sum_{n+1}^{\infty} m_{r} w_{r}+\sum_{r=0}^{n} m_{r} E_{r}
$$

Combining (5.11) and (5.6) we see that if $\eta_{0} / \eta_{1} \simeq 1$, our previous analysis holds. This will be true if the neglected terms $w_{r}(r \geqq n+1)$ are negligible, that is, if

$$
\sum_{n+1}^{\infty} m_{r} w_{r} \simeq 0, \text { and } \sum_{0}^{n} m_{r} E_{r} \simeq 0 \text {. }
$$

6. Conclusions. We have derived simply calculable strict bounds for the relative error in computing the most quickly increasing solution (for $r$ decreasing) of the three-term recurrence relation, using Miller's algorithm. These expressions provide a simple way of ascertaining a suitable starting value $r=n$, for prescribed relative error $E_{r}{ }^{*}$. In the cases considered the behaviour of $E_{r}{ }^{*}$ has also been analysed. For a given particular case, a short calculation should now be sufficient to determine either a suitable starting value, or a realistic upper bound for the error.

Acknowledgements. Part of this work was carried out while at the University of Oklahoma, Norman, and part at the Canadian Mathematical Congress (Summer 1966).

University of Alberta

Edmonton, Alberta, Canada

1. T. Fort, Finite Differences and Difference Equations in the Real Domain, Clarendon Press, Oxford, 1948. MR 9, 514.

2. A. Y. Khinchine, Continued Fractions, Noordhoff, Groningen, 1963. MR 28 \#5038.

3. A. N. Khovanski, The Application of Continued Fractions and Their Generalizations to Problems in Approximation Theory, Noordhoff, Groningen, 1963. MR 27 \#6058.

4. S. Makinouchi, Tech. Rep. Osaka Univ., No. 659, Vol. 15, Osaka, Japan.

5. J. C. P. Miller, "Bessel functions, Part II," in Mathematical Tables, Vol. X, British Assoc. Advancement Sci., Cambridge Univ. Press, New York, 1952.

6. F. W. J. Olver, "Error analysis of Miller's recurrence algorithm," Math. Comp., v. 18, 1964, pp. 65-74. MR 29 \#6656.

7. H. Shintani, J. Sci. Hiroshima Univ., Ser. A-I, v. 29, 1965, pp. 121-133.

8. I. A. Stegun \& M. Abramowitz, "Generation of Bessel functions on high speed computers," MTAC, v. 11, 1957, pp. 255-257. MR $20 \# 459$.

9. I. A. Stegun \& M. Abramowitz (Editors), Handbook of Mathematical Functions, National Bureau of Standards Appl. Math. Series, 55, U. S. Government Printing Office, Washington, D. C., 1964. MR $29 \# 4914$. 